\title{
Mesenchymal stem cell-like cells in classic renal angiomyolipoma
}

\author{
XINLONG YAN $^{1 *}$, LIXIN SHI $^{2 *}$, GUANGFU CHEN $^{2}$, XU ZHANG $^{2}$, BING LIU $^{3}$, \\ WEN YUE ${ }^{1}$, XUETAO PEI $^{1}$ and SHENGKUN SUN ${ }^{2}$ \\ ${ }^{1}$ Stem Cells and Regenerative Medicine Laboratory, Institute of Beijing Transfusion Medicine, Beijing 100850; \\ ${ }^{2}$ Department of Urology, PLA General Hospital, Beijing 100853; ${ }^{3}$ Department of Tumor Molecular Biology, \\ Affiliated Hospital to the Academy of Medical Sciences, Beijing 100071, P.R. China
}

Received February 19, 2012; Accepted May 31, 2012

DOI: $10.3892 / \mathrm{ol} .2012 .760$

\begin{abstract}
As a benign mesenchymal tumor, classic renal angiomyolipoma (AML) may obliterate the kidney parenchyma and cause renal hemorrhage. It has previously been reported that mesenchymal stem cells (MSCs) are involved in tumorigenesis; however, there have been no studies on stem cells with renal AML origin. In the present study, six females with classic renal AML received a partial or total nephrectomy. During surgery, tumor tissues were collected and culture expansion of adhesive fibroblastoid cells from these tissues was performed. We successfully isolated and cultured MSC-like cells from all six renal AML tumors. MSC characteristics, including morphology, immunophenotype and multidifferentiation potential were analyzed. Flow cytometry analysis revealed that these cells are highly similar to human bone marrow MSCs due to the expression of MSC-specific surface proteins, including CD29, CD44, CD73, CD90 and CD105. The stem cell-like nature of these cells is further supported by their adipogenic and osteogenic differentiation potentials when incubated in appropriate differentiation cocktails. Renal AML-derived adhesive cells possessing the characteristics of MSCs are described for the first time. They are a novel cell type which may be useful in future studies with regards to determining the role of stem cells in the formation and development of renal AML.
\end{abstract}

\section{Introduction}

Renal angiomyolipoma (AML) is a benign mesenchymal tumor composed of smooth muscle, adipose tissue and blood vessels. Although renal AML uniformly follows a benign course, these tumors are susceptible to provoking life threatening hemor-

Correspondence to: Professor Shengkun Sun, Department of Urology, PLA General Hospital, 28 Fuxing Road, Beijing 100853, P.R. China

E-mail: shengkunsun@301hospital.com.cn; saintkuns@gmail.com

*Contributed equally

Key words: renal angiomyolipoma, renal tumor, mesenchymal stem cells rhages and replacement of the kidney parenchyma, resulting in renal failure. To date, the only treatments for renal AML are excision or embolization.

Various hypotheses have been proposed to explain the tumorigenesis of renal AML; however, the cellular origin and histogenesis remain unknown. Lim et al hypothesized that the tumor originates from a pluripotent cell derived from the neural crest, which may give rise to smooth muscle cells and melonocytes (1). Bonetti et al suggested that lung AMLs are derived from distinctive perivascular epithelioid cells, a cell type of which no normal counterpart has been convincingly demonstrated (2). Barnard and Lajoie proposed that the cell of origin is a smooth muscle cell resembling a pericyte that exhibits unusual features, including melanocytic differentiation (3).

As a mesenchymal tumor, classic renal AML is histologically composed of smooth muscle, adipose tissue and thick blood vessel walls. This tripartite-tissue composition had lead us to the hypothesis that renal AML may arise from mesenchymal stem cells (MSCs). MSCs, which are present in adult bone marrow, are considered to be multipotent cells, and have the potential to differentiate into the full lineage of mesenchymal tissues, including bone, cartilage, fat, muscle and endothelial cells of blood vessels (4). It has been demonstrated that MSCs reside in the connective tissues of numerous organs, including normal and neoplastic kidneys (5-9). However, stem cell characteristics have not been studied in classic renal AML and the distribution of MSCs in renal AML remains unknown.

In this study, we aimed to verify this hypothesis by establishing a culture method to isolate MSC-like cells from classic renal AML. Further characterizations of these MSC-like cells were also confirmed in this study.

\section{Subjects and methods}

Subjects. A total of 6 female patients with classic renal AML underwent partial or radical nephrectomy between March 2009 and September 2010 at the PLA General Hospital, Beijing, China. The age of the patients ranged from 16-48 years, with an average age of $40.7 \pm 12.4$ years. The mean tumor diameter was $11.9 \pm 6.2 \mathrm{~cm}$. Classic AML was diagnosed radiographically based on the presence of fat, and histologically based on the presence of a combination of smooth muscle, adipose tissue and thick blood vessel walls. During surgery, renal AML 
tissues were obtained from each patient. Hematoxylin and eosin (H\&E) and immunohistochemical staining for $\alpha$-smooth muscle actin and HMB-45 was evaluated for each tissue section by a reporting pathologist to confirm the original diagnosis. The Ki67 protein was used as a marker to distinguish between the epithelioid variant of AML (Ki67-positive) and classic AML (Ki67-negative) (10). Informed consent was obtained from each patient prior to surgery and the study was approved by the Institutional Review Board of PLA General Hospital.

Isolation and primary cell culture of MSCs from renal AML. Fresh and sterile renal AML tissues were collected during surgery. The surface of the tumor tissues was removed and the inner parts were cut into $1-3 \mathrm{~mm}^{3}$-sized pieces. Once contaminating debris and red blood cells were removed using sterile phosphate-buffered saline (PBS), the tissues were minced using scalpels in a tissue culture dish. They were then enzymatically dissociated in $5 \mathrm{ml} 0.075 \%$ collagenase (type I; Sigma-Aldrich, St. Louis, MO, USA) in PBS for $30 \mathrm{~min}$ at $37^{\circ} \mathrm{C}$ with gentle agitation. The collagenase was inactivated using an equal volume of Dulbecco's modified Eagle's medium (DMEM) containing 10\% fetal bovine serum (FBS). A single-cell suspension was incubated in $\alpha$-minimun essential medium (MEM) without ribonucleosides and deoxyribonucleosides (Invitrogen, Carlsbad, CA, USA) containing 10\% selected FBS, 0.45 mM monothioglycerol (MTG; SigmaAldrich), 100 units/ml penicillin (Hyclone, Logan, UT, USA), $100 \mathrm{ng} / \mathrm{ml}$ streptomycin (Hyclone) and $1 \mathrm{ng} / \mathrm{ml} \mathrm{bFGF} \mathrm{(R \& D}$ Systems, Minneapolis, MN, USA). The medium was changed every 2 days and the adherent cells were harvested by trypsinization when $80-90 \%$ confluence was reached. The cells were then passaged at a ratio of 1:3 for further expansion.

Flow cytometry and immunofluorescence staining. Single-cell suspensions of MSCs were stained (11). Aliquots of 3x10 cells were labeled with fluorescein isothiocyanate (FITC)- or phycoerythrin (PE)-conjugated monoclonal antibodies against human CD14, CD19, CD29, CD31, CD34, CD44, CD73, CD105, CD144, CD166 and HLA-DR for $30 \mathrm{~min}$ at $4^{\circ} \mathrm{C}$. The cells were washed 3 times in cold PBS and analyzed using a BD FACSCalibur (BD Biosciences, San Jose, CA, USA). Antibodies recognizing CD14, CD19, CD34, CD73, CD105, CD166 and HLA-DR were purchased from BD Biosciences. Antibodies against CD29 and CD44 were purchased from BioLegend (San Diego, CA, USA), and antibodies against CD31 and CD144 were obtained from eBioscience (San Diego, CA, USA).

Adipogenic and osteogenic differentiation assay. For adipogenic differentiation, the cultures were incubated in high-glucose DMEM containing 10\% FBS, supplemented with $10 \%$ horse serum, $10^{-6} \mathrm{M}$ dexamethasone, $0.5 \mu \mathrm{M}$ isobutylmethylxanthine, $5 \mathrm{ng} / \mathrm{ml}$ insulin, $60 \mu \mathrm{M}$ indomethacin and $10^{-4} \mathrm{M}$ hydrocortisone (Sigma-Aldrich) for 1 week. Characterization of adipocytes was performed using Oil-Red-O staining. For osteogenic differentiation, cells were incubated in high-glucose DMEM containing 10\% FBS, supplemented with $0.2 \mathrm{mM}$ ascorbic acid, $10^{-7} \mathrm{M}$ dexamethasone and $10 \mathrm{mM} \beta$-glycerol phosphate (Sigma-Aldrich) for 2 weeks. The osteogenic differentiation was evaluated using alkaline phosphatase (ALP) staining.

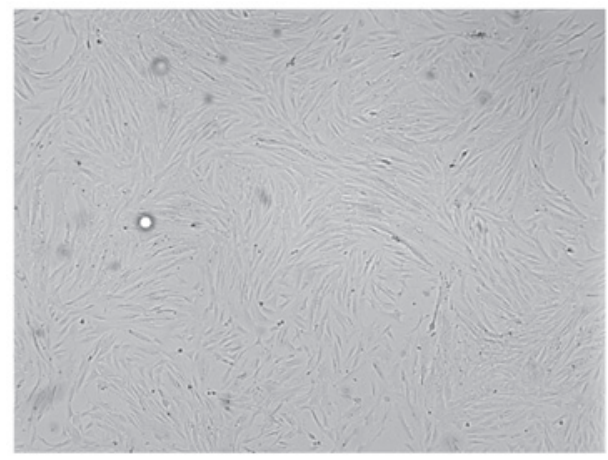

Figure 1. Morphology of MSC-like cells from renal AML. Adherent cells isolated from renal AML were long, spindle-shaped and fibroblastic in appearance (magnification, x100). MSC, mesenchymal stem cells; AML, angiomyolipoma.

\section{Results}

Diagnosis and development of cell culture from renal AML. Specimens from all 6 cases of human renal AML revealed uniform H\&E staining of the thick blood vessel walls and varying amounts of adipose tissue and smooth muscles. Immunohistochemical studies revealed uniform expression of the melanocytic markers HMB-45 and $\alpha$-smooth muscle actin. All sections were negative for Ki67, which confirmed classic renal AML.

Following 1 day of cell culture, the medium revealed that the majority of the cells or cell aggregates, including erythrocytes, were in the suspension of the minced AML tissue. The culture was then washed to remove these cells and few attached single cells or cell clumps were observed. Following 4 days in $25 \mathrm{~cm}^{2}$ flasks these attached cells actively proliferated and reached $80-90 \%$ confluency. The morphological observations demonstrated that these cells had a typical MSC-like appearance (Fig. 1).

Phenotype. Flow cytometry analyses were used to determine the immunophenotype of these putative AML-MSCs. We compared the expression levels of specific surface markers associated with stem cells. Cultured cells did not express CD14, CD31, CD34, CD45 or CD144; excluding the growth in the cultures of contaminating endothelial or hematopoietic cells. Cells expressed all commonly accepted MSC markers, including CD29, CD44, CD73, CD90 and CD105. Additionally, they were negative for HLA-DR (Fig. 2). It was demonstrated that these cells possessed a characteristic MSC immunophenotype, which parallels the phenotypic profile of human bone marrow derived-MSCs.

Induction of multilineage mesenchymal cell differentiation. To investigate the differentiation potential of these MSC-like cells from AML tissues, we cultured cells under conditions that favored osteogenic or adipogenic differentiation. When cultured in an adipogenic differentiation medium for 4 days, preadipocytes with bright lipid droplets appeared. The lipid vacuoles became larger following further induction and were clearly visible without staining under a phase microscope. Following Oil-Red-O staining, the lipid nature of these red 

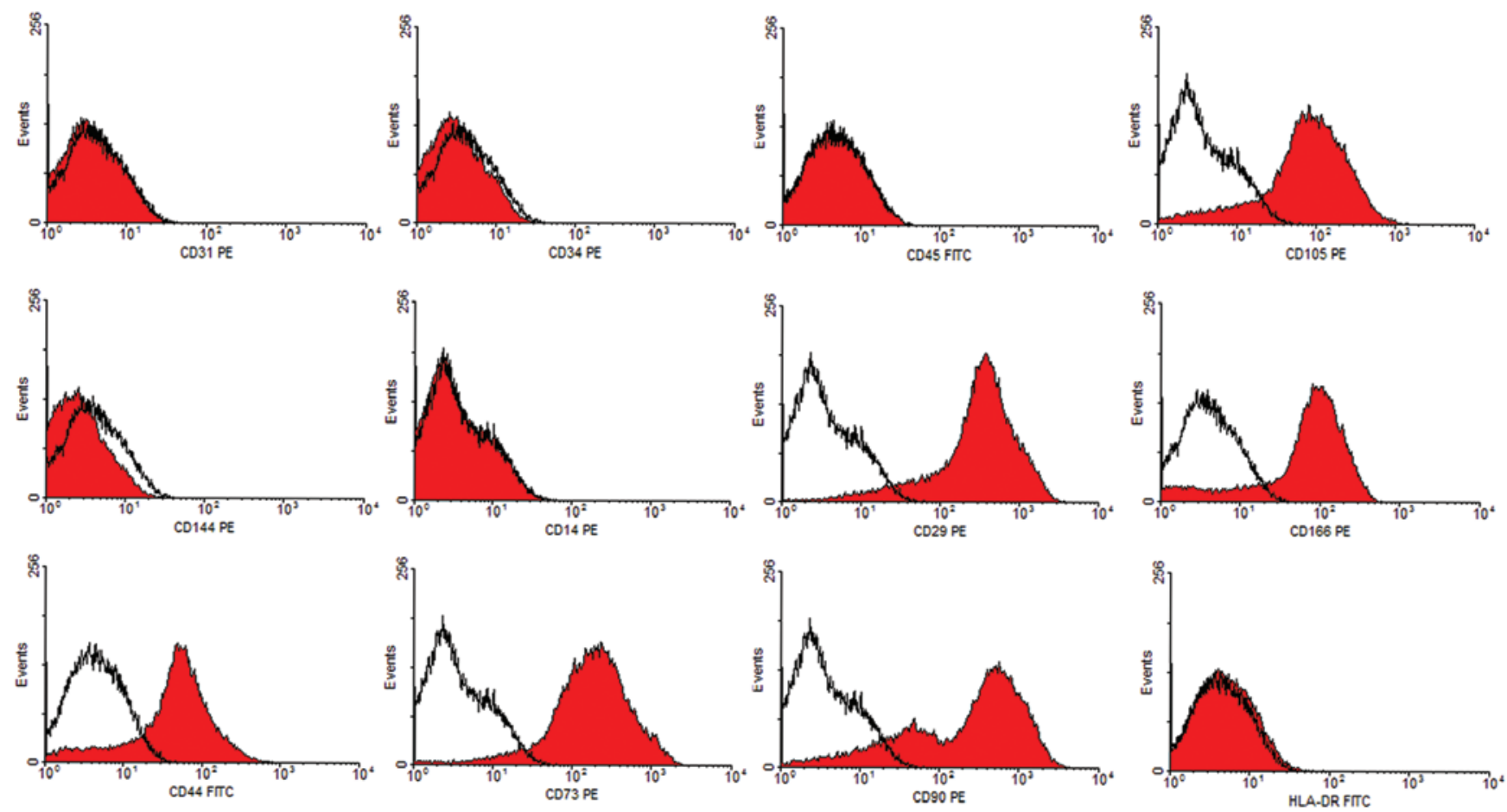

Figure 2. Surface antigens of MSC-like cells from renal AML. Representative FACS analysis demonstrated positivity for MSC markers CD29, CD44, CD73, CD90, CD105 and CD166, but negativity for CD14, CD31, CD34, CD45, CD144 and HLA-DR. Dark lines indicate the isotypic control. FITC, fluorescein isothiocyanate; PE, phycoerythrin. MSC, mesenchymal stem cells; AML, angiomyolipoma.


Figure 3. Induction of multilineage mescenchymal cell differentiation. (A-D) Adipogenesis. In the control group (without adipogenic differentiation), (A) no bright lipid droplets/vacuoles or (B) red stained cells were observed. Following adipogenic differentiation, (C) bright lipid droplets/vacuoles were clearly observed without staining and (D) cells demonstrated a positive reaction using Oil-Red-O staining. (E-H) Osteogenesis. (E and F) In the control group (without osteogenic differentiation), (F) ALP staining revealed only slight positivity. (G and H) Following osteogenic differentiation, (G) cells revealed a cuboidal morphology and $(\mathrm{H})$ ALP staining revealed strong positivity. ALP, alkaline phosphatase.

vacuoles was evident (Fig. 3). When cultured in osteogenic differentiation medium for 2 weeks, MSC-like cells differentiated into osteocytes, which stained positive for ALP (Fig. 3). Non-treated control cells revealed no significant spontaneous adipocyte or osteoblast formation.

\section{Discussion}

In this study, we successfully isolated adherent fibroblastoid cells from 6 cases of classic renal AML and investigated their biological characteristics. These AML-derived cells share a 
panel of surface antigens similar to MSCs isolated from other tissues, and are functionally able to differentiate into mesenchymal cells, including osteocytes and adipocytes.

MSCs have been isolated from various tumors, and have been suggested to contribute in human and mouse cancer models. Spontaneous malignant transformation has been reported in cultured murine MSCs, which transforms into osteosarcoma or fibrosarcoma. Malignant transformations have also been reported in long-term cultured human MSCs and are considered to play a key role in age-related tumors (12-15). Ewing's sarcoma was recently reported to be derived from MSCs, and cells isolated from this type of tumor tissue are able to differentiate along the adipogenic and osteogenic lineages (16). It is also reported that Wilms tumor cell lines are highly similar to human MSCs and express the MSC-specific surface proteins CD105, CD90 and CD73. The stem cell-like nature of the tumor cells is further supported by their adipogenic, chondrogenic and osteogenic differentiation potentials (17).

Although MSCs may be the cell origin of malignant tumors (18), MSCs in benign tumors have not been greatly studied. Additionally, no study has addressed the possible presence of stem cells in renal AML. As a member of the perivascular epithelioid cell tumors (PECOMAS) family, renal AML tumors are recognized as clonal neoplasms of the perivascular epithelial cells, which are mainly pericytes $(19,20)$. It has also been demonstrated that MSC-like cells originate from pericytes surrounding capillaries and microvessels. These cells, either freshly isolated or cultured over a long time, are indistinguishable from classic MSCs $(21,22)$. Previous studies have demonstrated the similarities between MSCs and pericytes; however, neither cell sorting nor stringent cell characterization has been performed to further support a correlation between these cell types, and the term pericyte has been used in its anatomic literal sense without any functional connotation (23). These pioneering studies suggest a perivascular origin for MSCs and other adult progenitor cells.

In the present study, the AML-derived cells demonstrated fibroblastoid morphology, plastic adherence and continuous expansion in vitro, which lead us to examine their MSC nature. Their ability to differentiate into adipocytes and osteoblasts when cultured in the appropriate differentiation medium, and their specific cell-surface phenotype labeling proved that they were MSC-like cells. Identification of MSCs in the renal AML tissue further supports the hypothesis that MSCs may be an important component of the tumor, and their presence may contribute to the development and formation of renal AML.

Several questions remain to be answered in future studies. Firstly, the source of MSCs in AML is unclear. Due to the similarities between AML MSC-like cells and bone marrow MSCs, it is reasonable to speculate that MSCs in renal AML may migrate from bone marrow and may have an intimate association with the formation of AML. Previous studies indicate that the MSC compartment extends throughout the body postnatally as a result of its perivascular location (7). Secondly, the abundance and localization of these cells in AML tissues has not yet been explored. Although cultured MSC-like cells have been functionally well-characterized, the identity and localization of the native MSCs ancestor in vivo remains elusive. Thirdly, it is unknown whether these MSC-like cells are likely to differentiate into the triphasic histology in AML, although they are bipotent in the in vitro study. It is also not elucidated whether these MSC-like cells, as well as their decedents, constitute the entity of the AML tumor. In our in vivo study, MSC-like cells only formed tumors within 3 months of grafting into the nude mice (data not shown). As a benign tumor, classic renal AML is associated with a slow and consistent growth rate $(0.088 \mathrm{~cm} /$ year $)(24)$. This biological characteristic explains the loss of the tumorigenic ability of these cells.

In conclusion, we present the first study of the existence of MSC-like cells isolated from fresh classic renal AML tissues. Cells derived from these tumors bear MSC markers and may be induced to differentiate along at least two mesenchymal lineages. Isolation and identification of MSC-like cells contributes to our knowledge of classic renal AML and suggests an MSC origin of renal AML. Targeting mesenchymal progenitors may open new opportunities for designing alternative therapeutic strategies against renal AML.

\section{Acknowledgements}

This study was supported by the National Natural Science Foundation of China (Grant No. 30600613).

\section{References}

1. Lim SD, Stallcup W, Lefkove B, Govindarajan B, Au KS, Northrup H, et al: Expression of the neural stem cell markers NG2 and L1 in human angiomyolipoma: are angiomyolipomas neoplasms of stem cells? Mol Med 13: 160-165, 2007.

2. Bonetti F, Pea M, Martignoni G, Doglioni C, Zamboni G, Capelli P, et al: Clear cell ('sugar') tumor of the lung is a lesion strictly related to angiomyolipoma - the concept of a family of lesions characterized by the presence of the perivascular epithelioid cells (PEC). Pathology 26: 230-236, 1994.

3. Barnard M and Lajoie G: Angiomyolipoma: immunohistochemical and ultrastructural study of 14 cases. Ultrastruct Pathol 25: 21-29, 2001.

4. Cao H, Xu W, Qian H, Zhu W, Yan Y, Zhou H, et al: Mesenchymal stem cell-like cells derived from human gastric cancer tissues. Cancer Lett 274: 61-71, 2009.

5. Bussolati B, Bruno S, Grange C, Ferrando U and Camussi G: Identification of a tumor-initiating stem cell population in human renal carcinomas. FASEB J 22: 3696-3705, 2008.

6. Gupta S, Verfaillie C, Chmielewski D, Kren S, Eidman K, Connaire J, et al: Isolation and characterization of kidney-derived stem cells. J Am Soc Nephrol 17: 3028-3040, 2006.

7. da Silva Meirelles L, Chagastelles PC and Nardi NB: Mesenchymal stem cells reside in virtually all post-natal organs and tissues. J Cell Sci 119: 2204-2213, 2006.

8. Plotkin MD and Goligorsky MS: Mesenchymal cells from adult kidney support angiogenesis and differentiate into multiple interstitial cell types including erythropoietin-producing fibroblasts. Am J Physiol Renal Physiol 291: F902-F912, 2006.

9. Humphreys BD and Bonventre JV: Mesenchymal stem cells in acute kidney injury. Annu Rev Med 59: 311-325, 2008.

10. Ooi SM, Vivian JB and Cohen RJ: The use of the Ki-67 marker in the pathological diagnosis of the epithelioid variant of renal angiomyolipoma. Int Urol Nephrol 41: 559-565, 2009.

11. Sun S, Guo Z, Xiao X, Liu B, Liu X, Tang PH, et al: Isolation of mouse marrow mesenchymal progenitors by a novel and reliable method. Stem Cells 21: 527-535, 2003.

12. Aractingi S, Kanitakis J, Euvrard S, Le Danff C, Peguillet I, Khosrotehrani K, et al: Skin carcinoma arising from donor cells in a kidney transplant recipient. Cancer Res 65: 1755-1760, 2005.

13. Barozzi P, Luppi M, Facchetti F, Mecucci C, Alù M, Sarid R, et al: Post-transplant Kaposi sarcoma originates from the seeding of donor-derived progenitors. Nat Med 9: 554-561, 2003. 
14. Houghton J, Stoicov C, Nomura S, Rogers AB, Carlson J, Li H, et al: Gastric cancer originating from bone marrow-derived cells. Science 306: 1568-1571, 2004

15. Li H, Fan X, Kovi RC, Jo Y, Moquin B, Konz R, et al: Spontaneous expression of embryonic factors and p53 point mutations in aged mesenchymal stem cells: a model of age-related tumorigenesis in mice. Cancer Res 67: 10889-10898, 2007.

16. Tirode F, Laud-Duval K, Prieur A, Delorme B, Charbord P and Delattre O: Mesenchymal stem cell features of Ewing tumors. Cancer Cell 11: 421-429, 2007.

17. Royer-Pokora B, Busch M, Beier M, Duhme C, de Torres C, Mora $\mathrm{J}$, et al: Wilms tumor cells with WT1 mutations have characteristic features of mesenchymal stem cells and express molecular markers of paraxial mesoderm. Hum Mol Genet 19: 1651-1668, 2010.

18. Li N, Yang R, Zhang W, Dorfman H, Rao P and Gorlick R: Genetically transforming human mesenchymal stem cells to sarcomas: changes in cellular phenotype and multilineage differentiation potential. Cancer 115: 4795-4806, 2009.
19. Varma S, Gupta S, Talwar J, Forte F and Dhar M: Renal epithelioid angiomyolipoma: a malignant disease. J Nephrol 24: 18-22, 2011.

20. Paradis V, Laurendeau I, Vieillefond A, Blanchet P, Eschwege P, Benoît $\mathrm{G}$, et al: Clonal analysis of renal sporadic angiomyolipomas. Hum Pathol 29: 1063-1067, 1998.

21. Crisan M, Yap S, Casteilla L, Chen CW, Corselli M, Park TS, et al: A perivascular origin for mesenchymal stem cells in multiple human organs. Cell Stem Cell 3: 301-313, 2008

22. Crisan M, Chen CW, Corselli M, Andriolo G, Lazzari L and Péault B: Perivascular multipotent progenitor cells in human organs. Ann NY Acad Sci 1176: 118-123, 2009.

23. Corselli M, Chen CW, Crisan M, Lazzari L and Péault B Perivascular ancestors of adult multipotent stem cells. Arterioscler Thromb Vasc Biol 30: 1104-1109, 2010.

24. Mues AC, Palacios JM, Haramis G, Casazza C, Badani K, Gupta M, et al: Contemporary experience in the management of angiomyolipoma. J Endourol 24: 1883-1886, 2010. 